\title{
Transient Flows of Newtonian Fluid through a Rectangular Microchannel with Slip Boundary
}

\author{
Benchawan Wiwatanapataphee, ${ }^{1}$ Yong Hong Wu, ${ }^{2}$ and Suharsono Suharsono ${ }^{3}$ \\ ${ }^{1}$ Department of Mathematics, Faculty of Science, Mahidol University, Bangkok 10400, Thailand \\ ${ }^{2}$ Department of Mathematics \& Statistics, Curtin University of Technology, Perth, WA 6845, Australia \\ ${ }^{3}$ Department of Mathematics, Lampung University, Lampung 35145, Indonesia
}

Correspondence should be addressed to Benchawan Wiwatanapataphee; benchawan.wiw@mahidol.ac.th

Received 12 March 2014; Accepted 22 May 2014; Published 29 June 2014

Academic Editor: Xinguang Zhang

Copyright (C) 2014 Benchawan Wiwatanapataphee et al. This is an open access article distributed under the Creative Commons Attribution License, which permits unrestricted use, distribution, and reproduction in any medium, provided the original work is properly cited.

\begin{abstract}
We study the transient flow of a Newtonian fluid in rectangular microchannels taking into account boundary slip. An exact solution is derived by using the separation of variables in space and Fourier series expansion in time. It is found that, for different forms of driving pressure field, the effect of boundary slip on the flow behavior is qualitatively different. If the pressure gradient is constant, the flow rate is almost linearly proportional to the slip parameter $\ell$ when $\ell$ is large; if the pressure gradient is in a waveform, as the slip parameter $\ell$ increases, the amplitude of the flow rate increases until approaching a constant value when $\ell$ becomes sufficiently large.
\end{abstract}

\section{Introduction}

In recent years, many researchers worldwide focus on the study of behavior of materials at micro- and nanoscales $[1,2]$, leading to the development of many biological and engineering systems and devices. Most of these systems and devices involve fluid flow in microchannels, called microflows [1, 3-7]. Typical examples include drug delivery systems [8], fuel cell devices, energy conversion, and biological sensing devices [9]. As the functional characteristics of these systems depend on the behavior of fluid flow in the systems, the study of microflows is important and has attracted more and more attention from the engineering and science communities in order to derive a better understanding of the mechanism of microflows and consequently better design and control of the devices and systems $[1,6,10]$.

The field equations governing the flow of Newtonian fluids are the continuity equation and the Navier-Stokes equations. These equations are subject to a set of boundary conditions. The no-slip boundary condition is used traditionally; namely, the tangential fluid velocity relative to the solid is zero on the fluid-solid interface [11]. However, recent molecular dynamic simulations and experiments in micrometer scale have shown that the fluid flow in microsystems is granular and slip may occur on the fluid-solid interface [10, 12-17]. Hence, for the study of microflow, it is important to take into account the boundary slip of fluids on the fluid-solid interfaces.

Over the last few decades, intensive research has been carried out to study various problems of fluid flow with the no-slip assumption or a slip boundary condition [1, 5, 17-33]. For flows under the no-slip assumption, exact solutions to many problems have been obtained and are available in the literature [11,34-37]. But for slip flow very few exact solutions are available in the literature. Exact solutions for the fluid flow in circular microtubes and circular microannuals with boundary slip have been derived and discussed in the papers $[17,25,28]$.

For microchannels with rectangular cross-section, a noslip solution has been obtained [38-43]. For the slip case steady state solution has also been obtained [15, 35, 44-48]. However, so far no exact solution has been derived for the transient flow of fluids through rectangular channel under pulsatile pressure. As many microsystems and devices have microchannels of rectangular cross-section, it is important to derive exact analytical solutions for the behavior of transient flow through rectangular microchannels with slip boundary. 


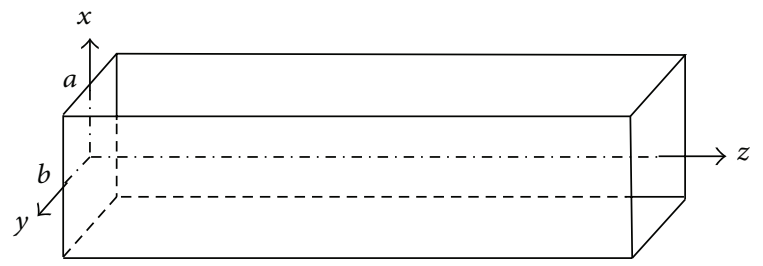

FIGURE 1: Rectangular microchannel.

Based on the current development in the field, in this paper, we study the time-dependent flow of incompressible Newtonian fluids through a rectangular microchannel taking into account boundary slip. The rest of the paper is organized as follows. In Section 2, we give the underlying initial boundary value problem for the transient slip flow. In Section 3, we derive the exact solution for the velocity field. In Section 4 , an analysis is conducted to study the effect of the slip length on the flow behavior. Finally, a conclusion is presented in Section 5 .

\section{Governing Boundary Value Problem}

Consider the unsteady flow of an incompressible Newtonian fluid in a rectangular channel of cross-section dimension $a \times b$ with the $z$-axes being in the axial direction as shown in Figure 1. The field equations governing the flow include the Navier-Stokes equations and the continuity equation. As the flow is symmetric about the $x z$-plane and the $y z$-plane and is fully developed, there is no cross-sectional flow and thus the velocity components in the $x$ and $y$ directions vanish; that is, $\vec{v}=\left(v_{x}, v_{y}, v_{z}\right)=(0,0, u)$.

Hence, from the continuity equation

$$
\frac{\partial v_{x}}{\partial x}+\frac{\partial v_{y}}{\partial y}+\frac{\partial v_{z}}{\partial z}=0
$$

and the Navier-Stokes equation

$$
\begin{aligned}
\rho\left(\frac{\partial v_{z}}{\partial t}+v_{x} \frac{\partial v_{z}}{\partial x}+v_{y} \frac{\partial v_{z}}{\partial y}+v_{z} \frac{\partial v_{z}}{\partial z}\right) \\
=-\frac{\partial p}{\partial z}+\mu\left(\frac{\partial^{2} v_{z}}{\partial x^{2}}+\frac{\partial^{2} v_{z}}{\partial y^{2}}+\frac{\partial^{2} v_{z}}{\partial z^{2}}\right)+\rho g_{z}
\end{aligned}
$$

the axial velocity, $u$, is governed by the following equation:

$$
\frac{\mu}{\rho}\left(\frac{\partial^{2} u}{\partial x^{2}}+\frac{\partial^{2} u}{\partial y^{2}}\right)-\frac{\partial u}{\partial t}=\frac{1}{\rho} \frac{\partial p}{\partial z} .
$$

As a large class of functions may be expressed by Fourier series, we consider, in this work, the flow of a fluid driven by the pressure gradient $\partial p / \partial z$ that may be expressed in the form of Fourier series given by

$$
\frac{\partial p}{\partial z}=a_{0}+\sum_{n=1}^{\infty}\left[a_{n} \cos (n \omega t)+b_{n} \sin (n \omega t)\right] .
$$

To define the problem completely, we supplement the field equation by the boundary condition. To take into account the boundary slip, the so-called Navier-slip boundary condition is used. On the fluid-solid interface $x= \pm a$ and $y= \pm b$, the axial fluid velocity, relative to the solid surface, is assumed to be proportional to the shear stress on the interface. Let $\mathbf{n}=\left(n_{1}, n_{2}, n_{3}\right)$ be the unit normal vector of the surface $S$ of the fluid, and let $\mathbf{t}=\left(t_{1}, t_{2}, t_{3}\right)$ be the positive tangential direction. Also let the fluid velocity on the wall direction be $v_{t}$, and let the velocity of the solid in the tangential direction of the surface be $v_{s t}$. Then, as shown in our previous work [17], the Navier-slip boundary condition can be expressed by

$$
\left(v_{t}-v_{s t}\right) t_{i}=-\frac{\ell\left(\sigma_{i j} n_{j} t_{i}\right)}{\mu}
$$

where the negative sign indicates that the surface traction force which acted on the fluid by the solid is opposite to the tangential velocity of fluid relative to the solid. For our problems in the $(x, y, z)$ coordinate system, $\mathbf{v}=(0,0, u)$ and $\mathbf{v}_{s}=(0,0,0)$. On the surface $x=a, \mathbf{t}=(0,0,1)$ and $\mathbf{n}=(1,0,0)$, and so $\left(v_{i}-v_{s}\right) t_{i}=v_{i} t_{i}=u$ and $\sigma_{i j} n_{j} t_{i}=$ $\sigma_{x z}=\mu(\partial u / \partial x)$ and consequently (5), on the surface $x=a$, becomes

$$
u(a, y, t)+\ell \frac{\partial u}{\partial x}(a, y, t)=0
$$

On the surface $x=-a$, we have $\mathbf{t}=(0,0,1)$ and $\mathbf{n}=(-1,0,0)$, and hence $\left(v_{i}-v_{s i}\right) t_{i}=v_{i} t_{i}=u$ and $\sigma_{i j} n_{j} t_{i}=-\sigma_{x z}=$ $-\mu(\partial u / \partial x)$ and consequently (5), on the surface $x=-a$, becomes

$$
u(-a, y, t)-\ell \frac{\partial u}{\partial x}(-a, y, t)=0
$$

Similarly, the boundary condition on the surface $y= \pm b$ is

$$
u(x, \pm b, t) \pm \ell \frac{\partial u}{\partial x}(x, \pm b, t)=0
$$

We will remark here that, for $\ell=0$, the slip boundary conditions (6)-(8) reduce to the no-slip condition; on the other extreme, where $\ell \rightarrow \infty$, (6)-(8) become the surface traction conditions for perfectly smooth surfaces; that is, $\sigma_{x z}( \pm a$, $y, t)=\sigma_{y z}(x, \pm b, t)=0$.

\section{Exact Solutions for Transient Velocity and Stress Fields}

To solve the partial differential equation (3), complex numbers are used to express the Fourier series for the pressure gradient; namely,

$$
\frac{\partial p}{\partial z}=\sum_{n=0}^{\infty}\left(a_{n} \cos (n \omega t)+b_{n} \sin (n \omega t)\right)=\operatorname{Re}\left(\sum_{n=0}^{\infty} c_{n} e^{i n \omega t}\right)
$$

where $c_{n}=a_{n}-b_{n} i$ and $e^{i n \omega t}=\cos (n \omega t)+i \sin (n \omega t)$. 
From the symmetry of the problem and the linearity of (3), we get $u=\sum_{n=0}^{\infty} \operatorname{Re}\left(u_{n}\right)$, where $u_{n}$ is defined by

$$
\begin{gathered}
\frac{\mu}{\rho}\left(\frac{\partial^{2} u_{n}}{\partial x^{2}}+\frac{\partial^{2} u_{n}}{\partial y^{2}}\right)-\frac{\partial u_{n}}{\partial t}=\frac{c_{n}}{\rho} e^{i n \omega t}, \\
\frac{\partial u_{n}}{\partial x}(0, y)=0, \quad \frac{\partial u_{n}}{\partial y}(x, 0)=0, \\
u_{n}(a, y, t)+\ell \frac{\partial u_{n}}{\partial x}(a, y, t)=0, \\
u_{n}(x, b, t)+\ell \frac{\partial u_{n}}{\partial y}(x, b, t)=0 .
\end{gathered}
$$

Through some mathematical analysis, we get that, for $n \geq 1$, the boundary value problem (10) has solution of the form

$$
u_{n}=f_{n}(x, y) e^{i n \omega t},
$$

where $f_{n}$ is determined by

$$
f_{n}=U_{n}(x, y)+V_{n}(x, y)-\frac{c_{n}}{i n \omega \rho},
$$

with $U_{n}(x, y)$ and $V_{n}(x, y)$ being governed, respectively, by the following boundary value problems:

$$
\begin{aligned}
& \text { BVP1: }\left\{\begin{array}{l}
\frac{\partial^{2} U_{n}}{\partial x^{2}}+\frac{\partial^{2} U_{n}}{\partial y^{2}}-i \frac{n \omega \rho}{\mu} U_{n}=0, \\
\frac{\partial U_{n}}{\partial x}(0, y)=0, \frac{\partial U_{n}}{\partial y}(x, 0)=0, \\
U_{n}(a, y)+\ell \frac{\partial U_{n}}{\partial x}(a, y)=\frac{c_{n}}{i n \omega \rho}, \\
U_{n}(x, b)+\ell \frac{\partial U_{n}}{\partial y}(x, b)=0,
\end{array}\right. \\
& \text { BVP2: }\left\{\begin{array}{l}
\frac{\partial^{2} V_{n}}{\partial x^{2}}+\frac{\partial^{2} V_{n}}{\partial y^{2}}-i \frac{n \omega \rho}{\mu} V_{n}=0, \\
\frac{\partial V_{n}}{\partial x}(0, y)=0, \frac{\partial V_{n}}{\partial y}(x, 0)=0, \\
V_{n}(a, y)+\ell \frac{\partial V_{n}}{\partial x}(a, y)=0, \\
V_{n}(x, b)+\ell \frac{\partial V_{n}}{\partial y}(x, b)=\frac{c_{n}}{i n \omega \rho} .
\end{array}\right.
\end{aligned}
$$

Thus, the remaining work for finding $f_{n}$ and consequently $u_{n}$ and then $u$ is to solve the two BVPs: BVP1 and BVP2. We first solve BVP1 by the separation of variables. From the PDE and the homogeneous boundary conditions of BVP1, we obtain

$$
U_{n}=\sum_{m=1}^{\infty} A_{n m} \cosh \left(\gamma_{n m} x\right) \cos \left(v_{m} y\right),
$$

where $v_{m}(m=1,2, \ldots)$ are the roots of the nonlinear equation

$$
\begin{gathered}
\cot (b v)=\ell v \\
\lambda_{m}=v_{m}^{2}, \quad \Phi_{m}=\cos \left(\sqrt{\lambda_{m}} y\right), \quad m=1,2,3, \ldots
\end{gathered}
$$

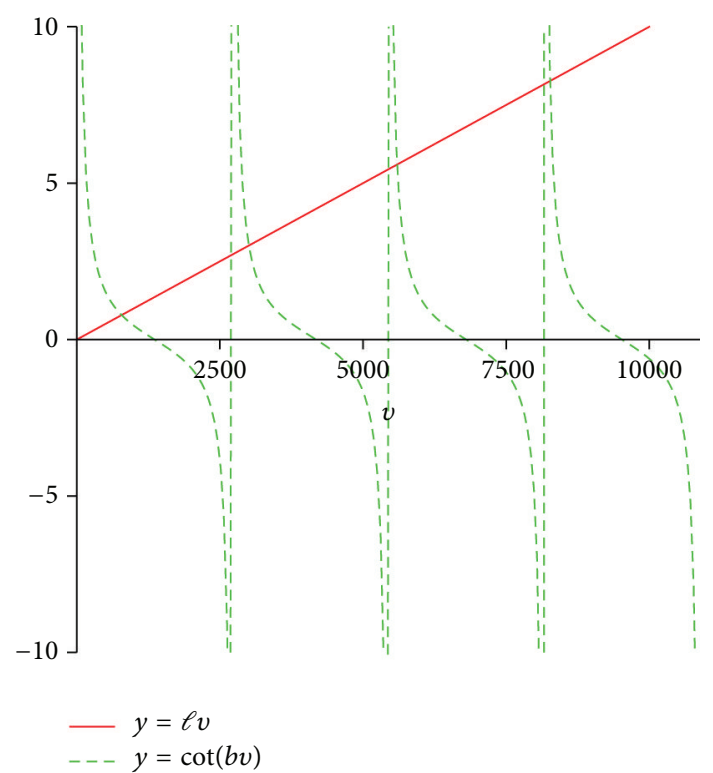

FIgURE 2: Infinite number of solutions of the two functions $y=\ell v$ and $y=\cot (b v)$.

are respectively eigenvalues and eigenfunctions of BVP1 and

$$
\gamma_{n m}=\sqrt{v_{m}^{2}+i \frac{n \rho \omega}{\mu}} .
$$

The eigenvalues $\lambda_{m}$ are the solution of the following nonlinear equation:

$$
\cot (\sqrt{\lambda} b)=\ell \sqrt{\lambda}
$$

which has infinite number of solutions as shown by the intersection of the graphs $y=\ell v$ and $y=\cot (b v)$ where $v=$ $\sqrt{\lambda}$ in Figure 2.

It can also be proved that the eigenfunctions $\Phi_{m}(m=$ $1,2, \ldots)$ are orthogonal and thus the coefficients $A_{n m}$ can be determined from the nonhomogeneous boundary condition of BVP1 by

$$
\begin{aligned}
& A_{n m} \\
& =-i \frac{4 c_{n} \sin \left(b v_{m}\right)}{n \rho \omega\left[2 b v_{m}+\sin \left(2 b v_{m}\right)\right]\left[\cosh \left(a \gamma_{n m}\right)+\ell \gamma_{n m} \sinh \left(a \gamma_{n m}\right)\right]} .
\end{aligned}
$$

Similarly, the solution $V_{n}$ of the BVP2 is

$$
V_{n}=\sum_{m=1}^{\infty} B_{n m} \cosh \left(\bar{\gamma}_{n m} y\right) \cos \left(\bar{v}_{m} x\right) \text {, }
$$


where $\bar{v}_{m}(m=1,2, \ldots)$ are the roots of the nonlinear equation

$$
\begin{gathered}
\cot (a \bar{v})=\ell \bar{v}, \\
\bar{\gamma}_{n m}=\sqrt{\bar{v}_{m}^{2}+i \frac{n \rho \omega}{\mu}}, \\
=-i \frac{4 c_{n} \sin \left(a \bar{v}_{m}\right)}{n \rho \omega\left[2 a \bar{v}_{m}+\sin \left(2 a \bar{v}_{m}\right)\right]\left[\cosh \left(b \bar{\gamma}_{m}+\ell \bar{\gamma}_{m n} \sinh \left(b \bar{\gamma}_{n m}\right)\right)\right]} .
\end{gathered}
$$

Substituting (14) and (20) into (12) yields the solution

$$
\begin{aligned}
& u_{n}(x, y, t) \\
& =e^{i n \omega t}\left\{i \frac{c_{n}}{n \rho \omega}\right. \\
& +\sum_{m=1}^{\infty}\left[A_{n m} \cosh \left(\gamma_{n m} x\right) \cos \left(v_{m} y\right)\right. \\
& \left.\left.+B_{n m} \cosh \left(\bar{\gamma}_{n m} y\right) \cos \left(\bar{v}_{m} x\right)\right]\right\}, \\
& n=1,2, \ldots .
\end{aligned}
$$

where $\gamma_{0 m}$ and $\bar{\gamma}_{0 m}$ are as defined in (17) and (22) with $n=0$; that is, $\gamma_{0 m}=v_{m}$ and $\bar{\gamma}_{0 m}=\bar{v}_{m} ; A_{0 m}$ and $B_{0 m}$ are as follows:

$$
\begin{aligned}
A_{0 m}=\left(-c_{0}[\right. & \left(a^{2}+2 a \ell\right) \sin \left(b v_{m}\right)+b^{2} \sin \left(b v_{m}\right) \\
& \left.+\frac{2}{v_{m}}\left(b \cos \left(b v_{m}\right)-\frac{\sin \left(b v_{m}\right)}{v_{m}}\right)\right] \\
\times(\mu & {\left[2 b v_{m}+\sin \left(2 b v_{m}\right)\right] } \\
\times & \left.\left.\times\left[\cosh \left(a v_{m}\right)+\ell v_{m} \sinh \left(a v_{m}\right)\right]\right)^{-1}\right),
\end{aligned}
$$

$$
\begin{aligned}
B_{0 m}=\left(-c_{0}[\right. & \left(b^{2}+2 b \ell\right) \sin \left(a \bar{v}_{m}\right)+a^{2} \sin \left(a \bar{v}_{m}\right) \\
& \left.+\frac{2}{\bar{v}_{m}}\left(a \cos \left(a \bar{v}_{m}\right)-\frac{\sin \left(a \bar{v}_{m}\right)}{\bar{v}_{m}}\right)\right] \\
\times & \left(\mu\left[2 a \bar{v}_{m}+\sin \left(2 a \bar{v}_{m}\right)\right]\right. \\
\times & \left.\left.\times\left[\cosh \left(b \bar{v}_{m}\right)+\ell \bar{v}_{m} \sinh \left(b \bar{v}_{m}\right)\right]\right)^{-1}\right) .
\end{aligned}
$$

Hence collecting all solutions of the subproblems, we have

$$
\begin{aligned}
& u(x, y, t) \\
& =\frac{a_{0}}{4 \mu}\left(x^{2}+y^{2}\right)+\sum_{n=1}^{\infty} \frac{-a_{n} \sin (n \omega t)+b_{n} \cos (n \omega t)}{n \omega \rho} \\
& +\sum_{n=0}^{\infty} \operatorname{Re}\left\{e^{i n \omega t}\right. \\
& \quad \times \sum_{m=1}^{\infty}\left[A_{m n} \cosh \left(\gamma_{n m} x\right) \cos \left(v_{m} y\right)\right. \\
& \left.\left.\quad+B_{n m} \cosh \left(\bar{\gamma}_{n m} y\right) \cos \left(\bar{v}_{m} x\right)\right]\right\},
\end{aligned}
$$

where $v_{m}$ and $\bar{v}_{m}$ are determined, respectively, by (15) and (21), $\gamma_{n m}$ and $\bar{\gamma}_{n m}$ are defined by (17) and (22), respectively, $A_{0 m}$ and $B_{n m}$ are defined by (26) and (27), and $A_{n m}$ and $B_{n m}(n, m \geq 1)$ are defined by (19) and (23), respectively.

Now we determine the exact solutions of the flow rate and the stresses in the fluid. From the velocity solution (28), we obtain the flow rate as follows:

$$
Q(t)=4 \int_{0}^{b} \int_{0}^{a} u(x, y, t) d x d y=\sum_{n=0}^{\infty} Q_{n}
$$

where $Q_{0}$ and $Q_{n}(n \neq 0)$ denote, respectively, the flow rate corresponding to the constant component and the nth harmonic component of the pressure gradient and

$$
\begin{aligned}
& Q_{0}=\frac{a_{0}}{3 \mu}\left[a^{3} b+a b^{3}\right] \\
&+4 \operatorname{Re} \sum_{m=1}^{\infty}\left\{\frac{A_{0 m}}{\gamma_{0 m} v_{m}} \sinh \left(a \gamma_{0 m}\right) \sin \left(b v_{m}\right)\right. \\
&\left.+\frac{B_{0 m}}{\bar{\gamma}_{0 m} \bar{v}_{m}} \sinh \left(b \bar{\gamma}_{0 m}\right) \sin \left(a \bar{v}_{m}\right)\right\},
\end{aligned}
$$




$$
\begin{aligned}
Q_{n}= & \frac{4 a b\left[-a_{n} \sin (n \omega t)+b_{n} \cos (n \omega t)\right]}{n \omega \rho} \\
+4 \operatorname{Re}\left\{e^{i n \omega t}\right. & \\
& \quad \times \sum_{m=1}^{\infty}\left[\frac{A_{n m}}{\gamma_{n m} v_{m}} \sinh \left(a \gamma_{n m}\right) \sin \left(b v_{m}\right)\right. \\
& \left.\left.+\frac{B_{n m}}{\bar{\gamma}_{n m} \bar{v}_{m}} \sinh \left(b \bar{\gamma}_{n m}\right) \sin \left(a \bar{v}_{m}\right)\right]\right\} .
\end{aligned}
$$

The stress tensor in the fluid can then be determined by the constitutive equation

$$
\sigma=-p \mathbf{I}+2 \mu \mathbf{d}
$$

where $\mathbf{I}$ is an identity matrix and the deformation rate $\mathbf{d}$ depends on the velocity by

$$
\mathbf{d}=\frac{1}{2}\left(\nabla v+(\nabla v)^{T}\right)
$$

As $\mathbf{v}=(0,0, u(x, y, t))$, we get

$$
d=\frac{1}{2}\left[\begin{array}{ccc}
0 & 0 & \frac{\partial u}{\partial x} \\
0 & 0 & \frac{\partial u}{\partial y} \\
\frac{\partial u}{\partial x} & \frac{\partial u}{\partial y} & 0
\end{array}\right]
$$

From (28) and (32)-(34), we obtain $d_{x x}=d_{y y}=d_{z z}=d_{x y}=$ 0 and

$$
\begin{aligned}
& d_{x z} \\
& =\frac{a_{0}}{2 \mu} x \\
& \quad+\frac{1}{2} \sum_{n=0}^{\infty} \operatorname{Re}\left\{e^{i n \omega t}\right. \\
& \quad \times \sum_{m=1}^{\infty}\left[A_{n m} \gamma_{n m} \sinh \left(\gamma_{n m} x\right) \cos \left(v_{m} y\right)\right. \\
& \left.\left.\quad-B_{n m} \bar{v}_{m} \cosh \left(\bar{\gamma}_{n m} y\right) \sin \left(\bar{v}_{m} x\right)\right]\right\},
\end{aligned}
$$

$$
\begin{aligned}
& d_{y z} \\
& =\frac{a_{0}}{2 \mu} y \\
& +\frac{1}{2} \sum_{n=0}^{\infty} \operatorname{Re}\left\{e^{i n \omega t}\right. \\
& \quad \times \sum_{m=1}^{\infty}\left[-A_{n m} v_{m} \cosh \left(\gamma_{n m} x\right) \sin \left(v_{m} y\right)\right. \\
& \left.\left.\quad+B_{n m} \bar{\gamma}_{n m} \sinh \left(\bar{\gamma}_{n m} y\right) \cos \left(\bar{v}_{m} x\right)\right]\right\} .
\end{aligned}
$$

Thus, from the constitutive equation (32), we get

$$
\begin{gathered}
\sigma_{x x}=\sigma_{y y}=\sigma_{z z}=-p=p_{0}(t)+q(t) z, \\
\sigma_{x y}=0, \quad \sigma_{x z}=2 \mu d_{x z}, \quad \sigma_{y z}=2 \mu d_{y z},
\end{gathered}
$$

where $q(t)$ denotes the pressure gradient $\partial p / \partial z$ while $p_{0}(t)$ is arbitrary and may be chosen to meet certain pressure condition.

\section{Investigation of the Effect of Boundary Slip on the Flow Behavior}

Based on the exact solutions obtained, we investigate the flow behaviour and the influence of the slip length in this section. As a general pressure field can be expressed by a Fourier series in the form of (4), from the principle of superposition, the exact solution of the problem is the superposition of the solutions corresponding to the constant pressure gradient plus the solutions corresponding to the sine or cosine waveform pressure gradients. In this work, without loss of generality, we consider flow problems under two different cases of driving pressure fields including the case with a constant pressure gradient and the case with a sine waveform pressure gradient. For simplicity, we introduce four dimensionless variables as follows:

$$
\varepsilon=\frac{b}{a}, \quad x^{*}=\frac{x}{a}, \quad y^{*}=\frac{y}{b}, \quad t^{*}=\frac{\omega t}{2 \pi} .
$$

Case $1\left(\partial p / \partial z=a_{0}\right)$. For this case, $c_{0}=a_{0}$ and $c_{n}=0$ for $n \geq 1$. The constant pressure gradient means that the pressure gradient does not depend on time. From (28), (30), (35), and (36), we get the following normalized velocity, flow rate, and shear stresses:

$$
\begin{aligned}
& u^{*}\left(x^{*}, y^{*}\right) \\
& =\frac{4 \mu}{a_{0} a^{2}} u=x^{* 2}+\left(\varepsilon y^{*}\right)^{2} \\
& \quad+\frac{4 \mu}{a_{0} a^{2}} \operatorname{Re} \sum_{m=1}^{\infty}\left[A_{0 m} \cosh \left(a v_{m} x^{*}\right) \cos \left(b v_{m} y^{*}\right)\right. \\
& \left.\quad+B_{0 m} \cosh \left(b \bar{v}_{m} y^{*}\right) \cos \left(a \bar{v}_{m} x^{*}\right)\right],
\end{aligned}
$$


$Q_{0}^{*}$

$$
\begin{aligned}
& =\frac{3 \mu}{a_{0} \varepsilon a^{4}} Q_{0}=1+\varepsilon^{2} \\
& +\frac{12 \mu}{a_{0} \varepsilon a^{4}} \operatorname{Re} \sum_{m=1}^{\infty}\left[\frac{A_{0 m}}{v_{m}^{2}} \sinh \left(a v_{m}\right) \sin \left(b v_{m}\right)\right. \\
& \left.+\frac{B_{0 m}}{\bar{v}_{m}^{2}} \sinh \left(b \bar{v}_{m}\right) \sin \left(a \bar{v}_{m}\right)\right],
\end{aligned}
$$

$$
\sigma_{x z}^{*}
$$

$$
\begin{aligned}
& =\frac{1}{a_{0} a} \sigma_{x z}=x^{*} \\
& +\frac{\mu}{a_{0} a} \operatorname{Re} \sum_{m=1}^{\infty}\left[A_{0 m} v_{m} \sinh \left(v_{m} a x^{*}\right) \cos \left(b v_{m} y^{*}\right)\right. \\
& \left.\quad-B_{0 m} \bar{v}_{m} \cosh \left(b \bar{v}_{m} y^{*}\right) \sin \left(a \bar{v}_{m} x^{*}\right)\right],
\end{aligned}
$$

$$
\begin{aligned}
& \sigma_{y z}^{*} \\
& \begin{aligned}
\frac{1}{a_{0} a} \sigma_{y z}=\varepsilon y^{*} \\
+\frac{\mu}{a_{0} a} \operatorname{Re} \sum_{m=1}^{\infty}\left[-A_{0 m} v_{m} \cosh \left(v_{m} a x^{*}\right) \sin \left(b v_{m} y^{*}\right)\right. \\
\left.\quad+B_{0 m} \bar{v}_{m} \sinh \left(b \bar{v}_{m} y^{*}\right) \cos \left(a \bar{v}_{m} x^{*}\right)\right] .
\end{aligned}
\end{aligned}
$$

As the pressure gradient does not depend on time, the normalized velocity and flow rate as well as stresses are influenced by the slip length $\ell$ only, which is implicitly contained in $v_{m}$ and $\bar{v}_{m}$.

Case $2\left(\partial p / \partial z=b_{1} \sin (\omega t)\right)$. For this case, the pressure gradient is sinusoidal with amplitude $b_{1}$, and $a_{0}=0, c_{1}=-b_{1} i$, $c_{n}=0$ for $\forall n \geq 2$. From (27), we have

$$
\begin{aligned}
& u^{*}\left(x^{*}, y^{*}\right) \\
& =\frac{\rho \omega}{b_{1}} u=\cos \left(2 \pi t^{*}\right) \\
& +\frac{\rho \omega}{b_{1}} \operatorname{Re} \sum_{m=1}^{\infty} e^{i 2 \pi t^{*}}\left[A_{1 m} \cosh \left(a \gamma_{1 m} x^{*}\right) \cos \left(b v_{m} y^{*}\right)\right. \\
& \left.+B_{1 m} \cosh \left(b \bar{\gamma}_{1 m} y^{*}\right) \cos \left(a \bar{v}_{m} x^{*}\right)\right] .
\end{aligned}
$$

Let

$$
\gamma_{1 m}=\left[v_{m}^{2}+i \frac{\rho \omega}{\mu}\right]^{1 / 2}:=\alpha_{1 m}+i \beta_{1 m}
$$

where

$$
\begin{gathered}
\alpha_{1 m}=\left[v_{m}^{4}+\left(\frac{\rho \omega}{\mu}\right)^{2}\right]^{1 / 4} \cos \left(\frac{\theta_{m}}{2}\right), \\
\beta_{1 m}=\left[v_{m}^{4}+\left(\frac{\rho \omega}{\mu}\right)^{2}\right]^{1 / 4} \sin \left(\frac{\theta_{m}}{2}\right), \\
\theta_{m}=\arctan \left(\frac{\rho \omega}{\mu v_{m}^{2}}\right) .
\end{gathered}
$$

Then

$$
\begin{aligned}
\cosh \left(a \gamma_{1 m}\right)= & \cosh \left(a \alpha_{1 m}\right) \cos \left(a \beta_{1 m}\right) \\
& +i \sinh \left(a \alpha_{1 m}\right) \sin \left(a \beta_{1 m}\right), \\
\sinh \left(a \gamma_{1 m}\right)= & \sinh \left(a \alpha_{1 m}\right) \cos \left(a \beta_{1 m}\right) \\
& +i \cosh \left(a \alpha_{1 m}\right) \sin \left(a \beta_{1 m}\right) .
\end{aligned}
$$

Using (19) and (23), through a lengthy derivation, we obtain

$$
u^{*}=\cos \left(2 \pi t^{*}\right)+\sum_{m=1}^{\infty}\left[d_{3 m} \cos \left(2 \pi t^{*}\right)-d_{4 m} \sin \left(2 \pi t^{*}\right)\right],
$$

where

$$
\begin{aligned}
& d_{1 m}=\cosh \left(a \alpha_{1 m}\right) \cos \left(a \beta_{1 m}\right) \\
& +\ell \alpha_{1 m} \sinh \left(a \alpha_{1 m}\right) \cos \left(a \beta_{1 m}\right) \\
& -\ell \beta_{1 m} \cosh \left(a \alpha_{1 m}\right) \sin \left(a \beta_{1 m}\right) \text {, } \\
& d_{2 m}=\sinh \left(a \alpha_{1 m}\right) \sin \left(a \beta_{1 m}\right) \\
& +\ell \alpha_{1 m} \cosh \left(a \alpha_{1 m}\right) \sin \left(a \beta_{1 m}\right) \\
& +\ell \beta_{1 m} \sinh \left(a \alpha_{1 m}\right) \cos \left(a \beta_{1 m}\right) \text {, } \\
& d_{3 m}=A_{1 m}^{*} \cos \left(b v_{m} y^{*}\right) \\
& \times\left[d_{1 m} \cosh \left(a \alpha_{1 m} x^{*}\right) \cos \left(a \beta_{1 m} x^{*}\right)\right. \\
& \left.+d_{2 m} \sinh \left(a \alpha_{1 m} x^{*}\right) \sin \left(a \beta_{1 m} x^{*}\right)\right] \\
& +B_{1 m}^{*} \cos \left(a \bar{v}_{m} x^{*}\right) \\
& \times\left[\bar{d}_{1 m} \cosh \left(b \bar{\alpha}_{1 m} y^{*}\right) \cos \left(b \bar{\beta}_{1 m} y^{*}\right)\right. \\
& \left.+\bar{d}_{2 m} \sinh \left(b \bar{\alpha}_{1 m} y^{*}\right) \sin \left(b \bar{\beta}_{1 m} y^{*}\right)\right], \\
& d_{4 m}=A_{1 m}^{*} \cos \left(b v_{m} y^{*}\right) \\
& \times\left[d_{1 m} \sinh \left(a \alpha_{1 m} x^{*}\right) \sin \left(a \beta_{1 m} x^{*}\right)\right. \\
& \left.-d_{2 m} \cosh \left(a \alpha_{1 m} x^{*}\right) \cos \left(a \beta_{1 m} x^{*}\right)\right] \\
& +B_{1 m}^{*} \cos \left(a \bar{v}_{m} x^{*}\right) \\
& \times\left[\bar{d}_{1 m} \sinh \left(b \bar{\alpha}_{1 m} y^{*}\right) \sin \left(b \bar{\beta}_{1 m} y^{*}\right)\right. \\
& \left.-\bar{d}_{2 m} \cosh \left(b \bar{\alpha}_{1 m} y^{*}\right) \cos \left(b \bar{\beta}_{1 m} y^{*}\right)\right], \\
& A_{1 m}^{*}=-\frac{4 \sin \left(b v_{m}\right)}{\left[2 b v_{m}+\sin \left(2 b v_{m}\right)\right]\left(d_{1 m}^{2}+d_{2 m}^{2}\right)}, \\
& A_{1 m}=\frac{b_{1}}{\rho \omega} A_{1 m}^{*}\left(d_{1 m}-d_{2 m} i\right), \\
& B_{1 m}^{*}=-\frac{4 \sin \left(a \bar{v}_{m}\right)}{\left[2 a \bar{v}_{m}+\sin \left(2 a \bar{v}_{m}\right)\right]\left(\bar{d}_{1 m}^{2}+\bar{d}_{2 m}^{2}\right)}, \\
& B_{1 m}=\frac{b_{1}}{\rho \omega} B_{1 m}^{*}\left(\bar{d}_{1 m}-\bar{d}_{2 m} i\right) \text {. }
\end{aligned}
$$




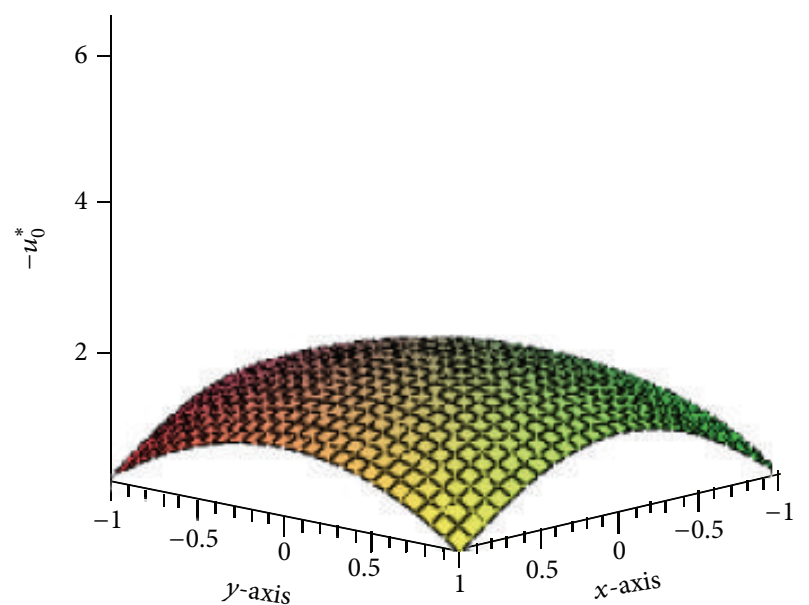

(a) $\ell=0.001$

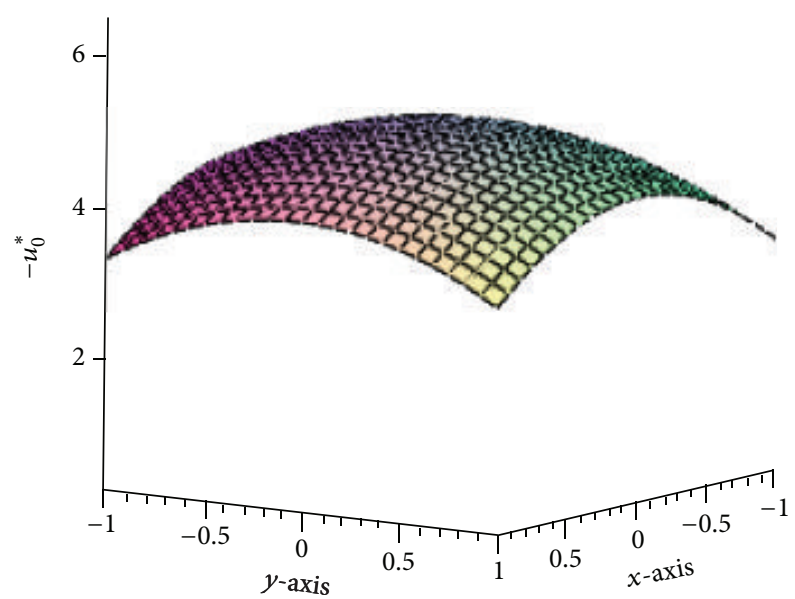

(c) $\ell=0.003$

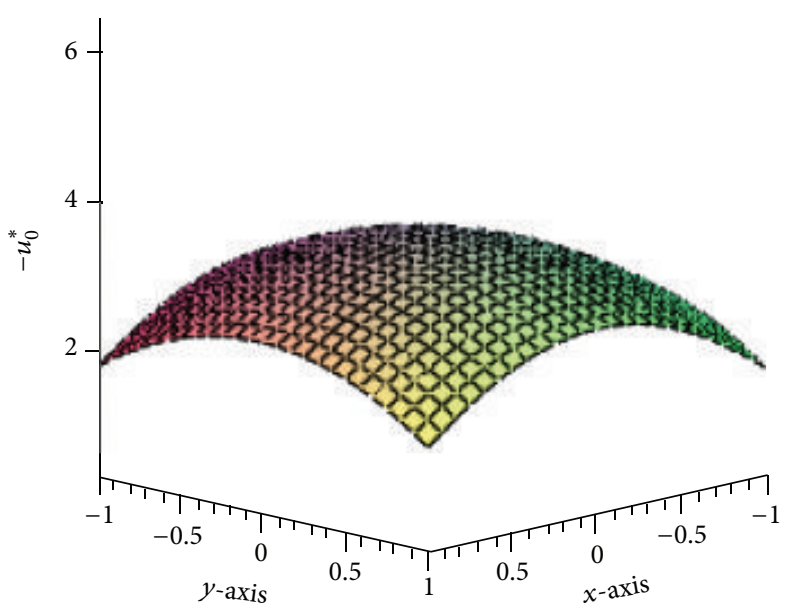

(b) $\ell=0.002$

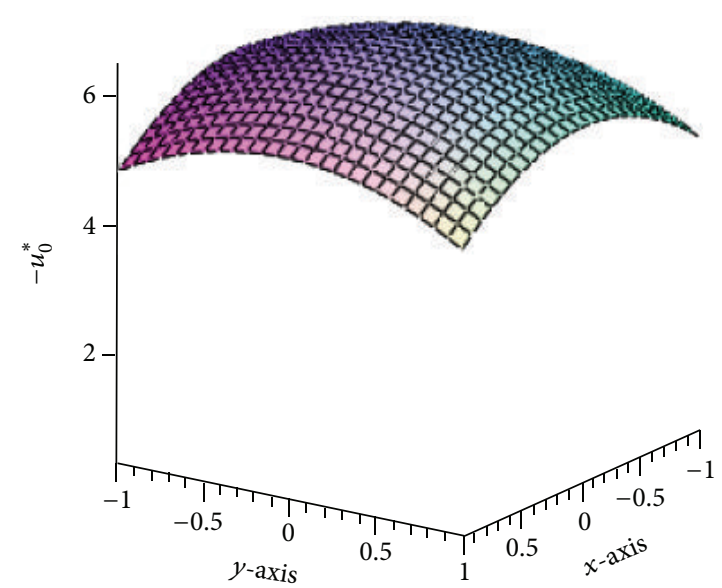

(d) $\ell=0.004$

FIgURE 3: Axial velocity profile on the cross-section of the channel with the area of $1.0 \times 10^{-6} \mathrm{~m}^{2}$ and ratio $\varepsilon$ of $3 / 4$ for constant pressure gradient under various values of slip lengths $\ell$ : (a) $\ell=0.001$; (b) $\ell=0.002$; (c) $\ell=0.003$; (d) $\ell=0.004$.

For convenience in discussion, transform (47) into the following form:

$$
u^{*}=u_{m}^{*} \cos \left(2 \pi t^{*}+\theta_{u}\right)
$$

where $u_{m}^{*}$ and $\theta_{u}$ denote the amplitude and phase angles of the normalized velocity defined, respectively, by

$$
\begin{gathered}
u_{m}^{*}=\left[\left(1+\sum_{m=1}^{\infty} d_{3 m}\right)^{2}+\left(\sum_{m=1}^{\infty} d_{4 m}\right)^{2}\right]^{1 / 2}, \\
\theta_{u}=\arctan \left(\frac{\sum_{m=1}^{\infty} d_{4 m}}{1+\sum_{m=1}^{\infty} d_{3 m}}\right) .
\end{gathered}
$$

The flow rate is

$$
\begin{aligned}
Q_{1}= & \frac{4 a b b_{1} \cos \left(2 \pi t^{*}\right)}{\rho \omega} \\
+4 \sum_{m=1}^{\infty} \operatorname{Re}\left(e^{i 2 \pi t^{*}}\right. & \\
& \quad \times\left(\frac{A_{1 m}}{\gamma_{1 m} \nu_{m}} \sinh \left(a \gamma_{1 m}\right) \sin \left(b \nu_{m}\right)\right. \\
& \left.\left.+\frac{B_{1 m}}{\bar{\gamma}_{1 m} \bar{\nu}_{m}} \sinh \left(b \bar{\gamma}_{1 m}\right) \sin \left(a \bar{\nu}_{m}\right)\right)\right) .
\end{aligned}
$$




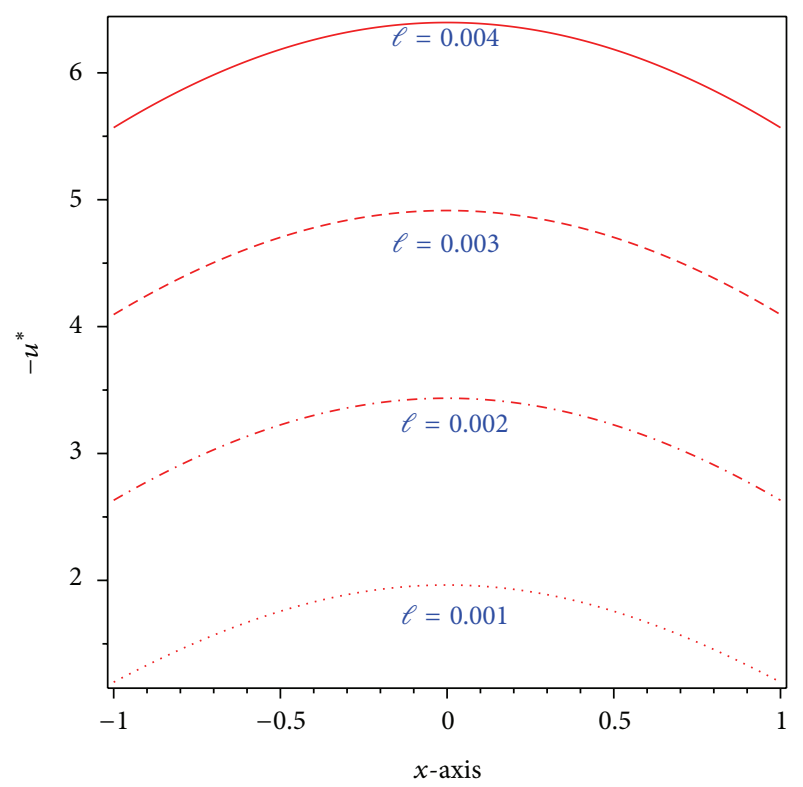

(a)

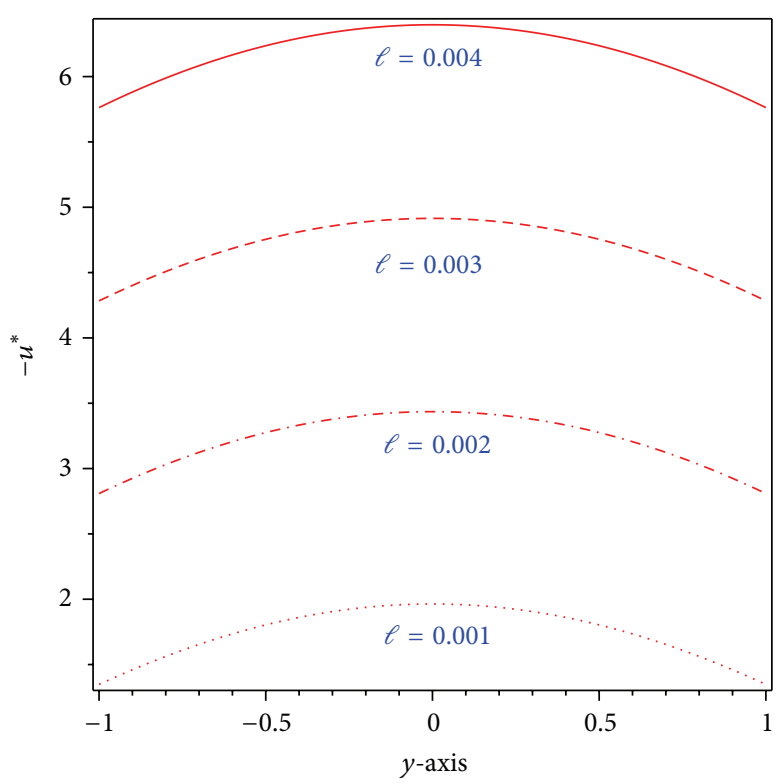

(b)

FIGURE 4: 2D velocity profile along the $x$-axis and $y$-axis on the cross-section of the channel with the area of $1.0 \times 10^{-6} \mathrm{~m}^{2}$ and ratio $\varepsilon$ of $3 / 4$ for constant pressure gradient under different slip lengths $\ell$ values.

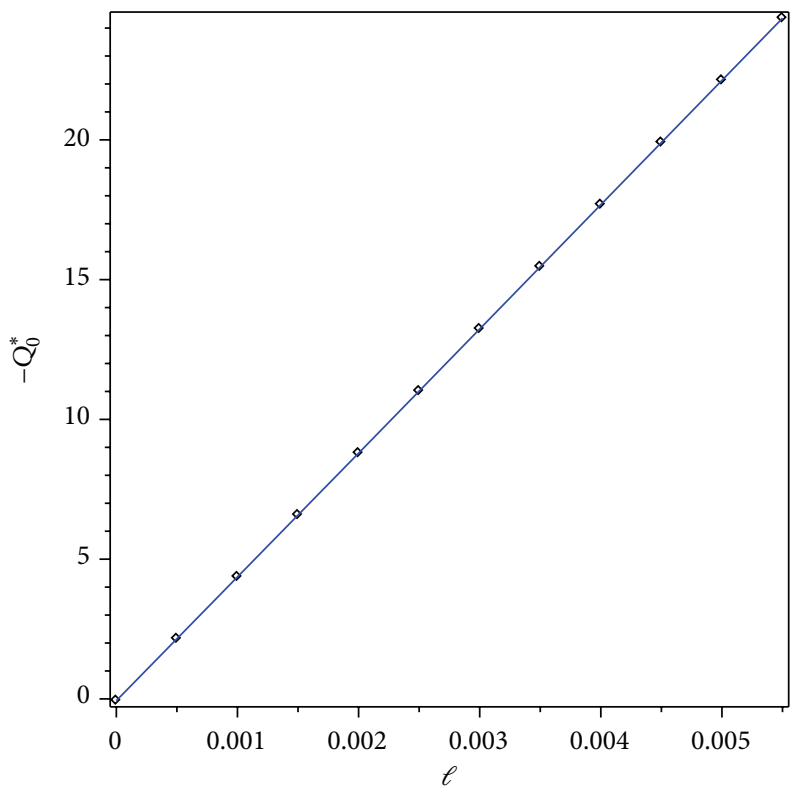

(a)

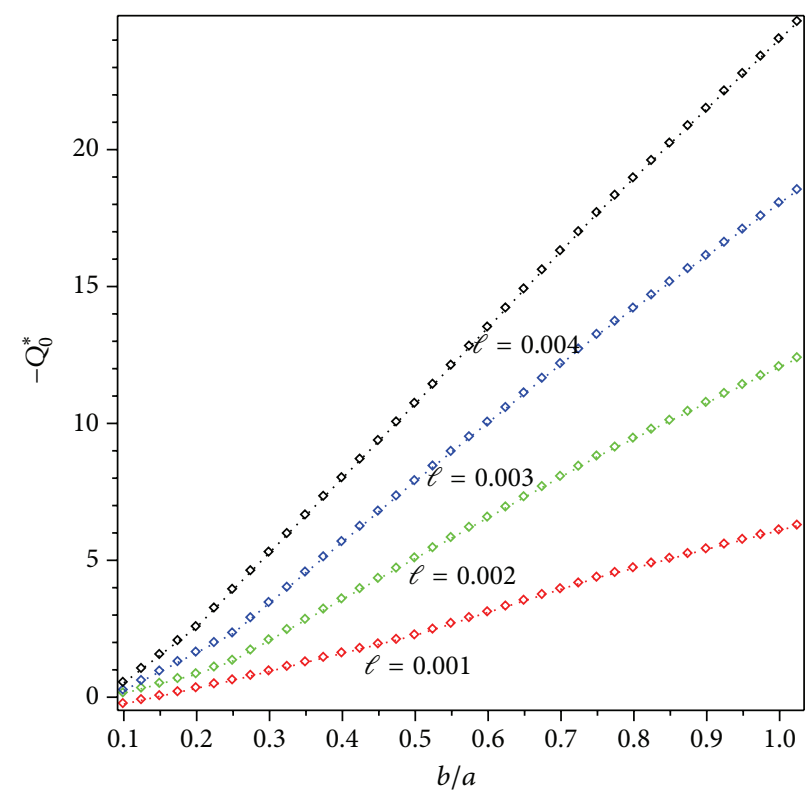

(b)

FIGURE 5: Influence of slip length and the ratio $\varepsilon$ on the flow rate $Q_{0}^{*}$ on the cross-section of the channel with the constant area of $1.0 \times 10^{-6} \mathrm{~m}^{2}$ for a constant gradient pressure. 


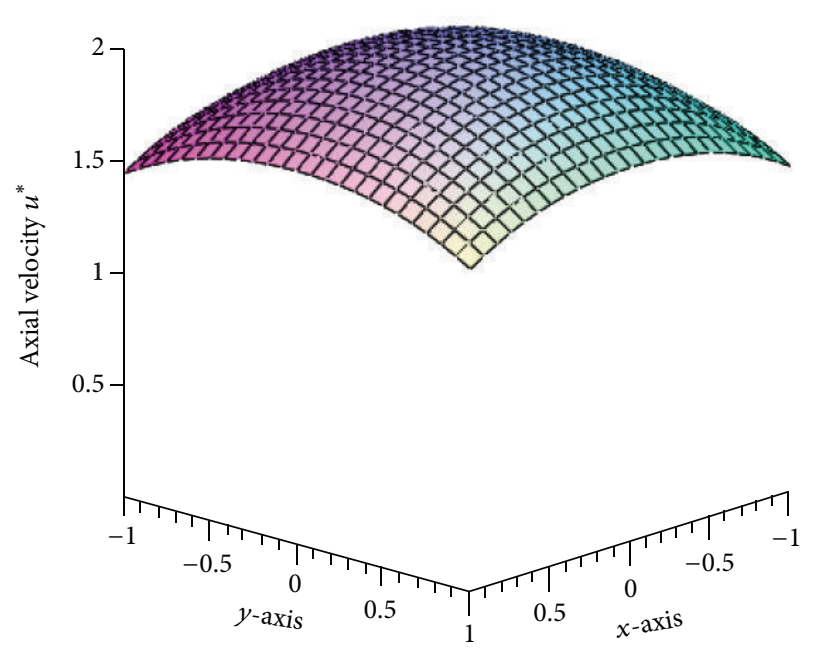

(a) $t^{*}=0$

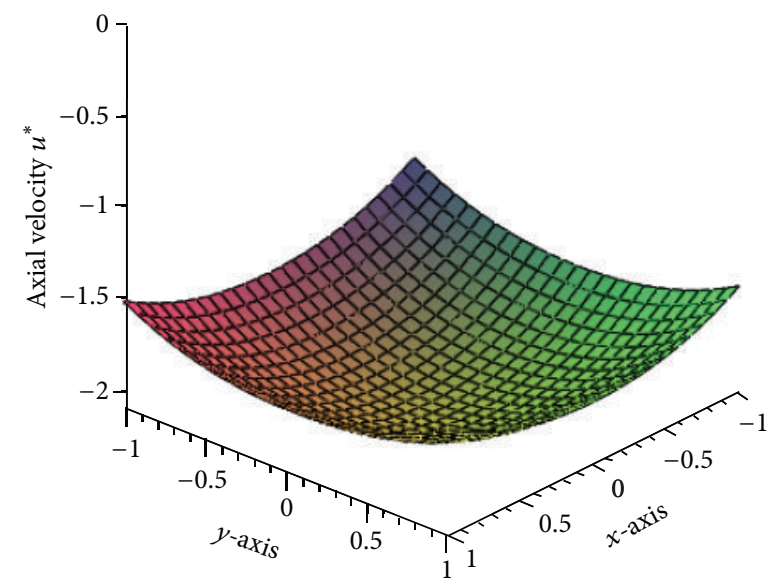

(c) $t^{*}=0.5$

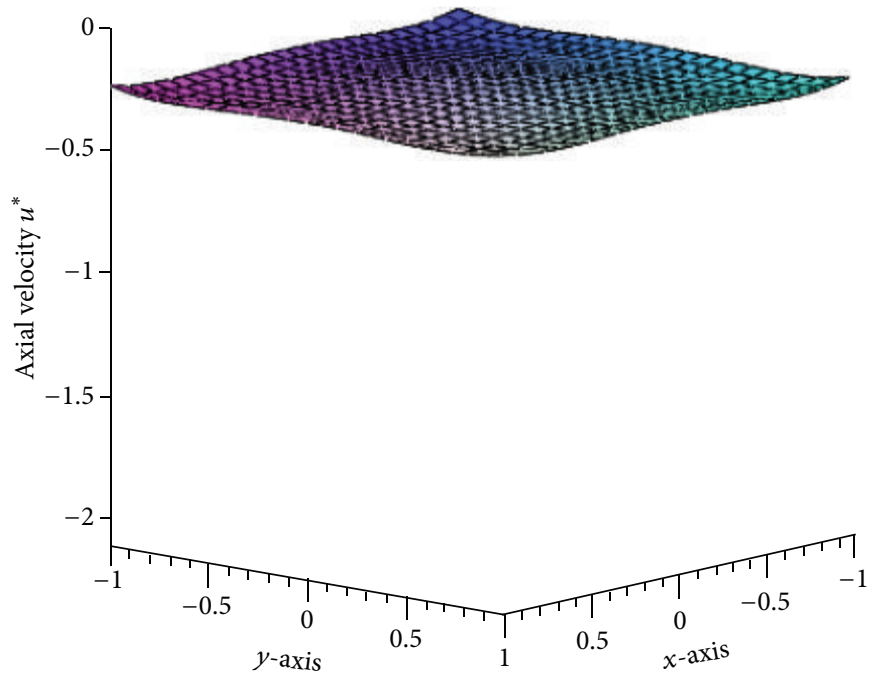

(b) $t^{*}=0.25$

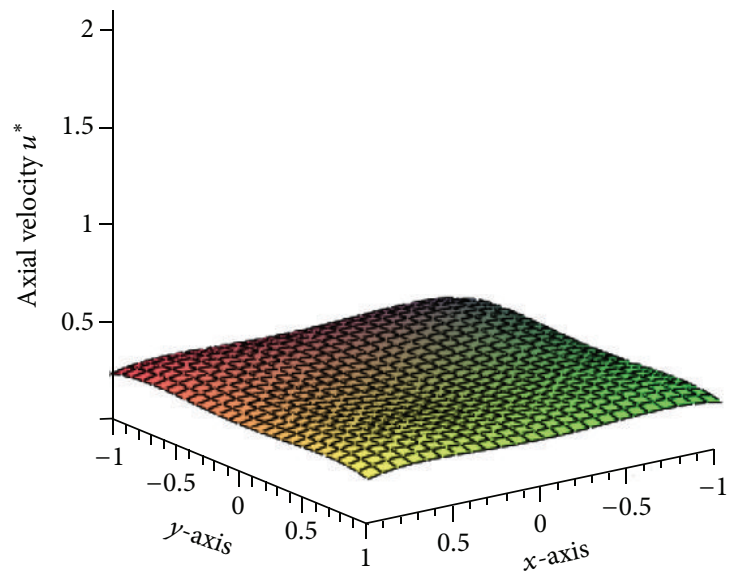

(d) $t^{*}=0.75$

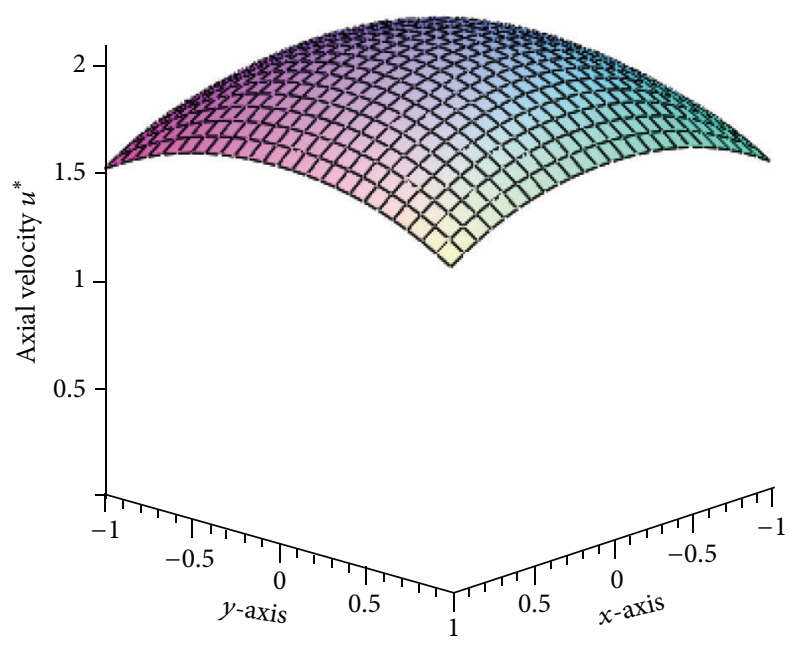

(e) $t^{*}=1$

Figure 6: Axial velocity profile on the cross-section of the channel with the area of $1.0 \times 10^{-6} \mathrm{~m}^{2}$ and ratio $b / a=1$ under the frequency $\omega=\alpha / \rho a^{2}$ with $\alpha=0.005$ and slip length of 0.001 for various instants of time. 
Using real arithmetic, through lengthy calculation, we obtain the normalized flow rate

$$
\begin{aligned}
Q_{1}^{*}= & \frac{\rho \omega}{b_{1}(4 a b)} Q_{1}=\cos \left(2 \pi t^{*}\right) \\
& +\frac{1}{a b} \sum_{m=1}^{\infty}\left[d_{5 m} \cos \left(2 \pi t^{*}\right)-d_{6 m} \sin \left(2 \pi t^{*}\right)\right] \\
= & {\left[1+\frac{1}{a b} \sum_{m=1}^{\infty} d_{5 m}\right] \cos \left(2 \pi t^{*}\right) } \\
& -\left[\frac{1}{a b} \sum_{m=1}^{\infty} d_{6 m}\right] \sin \left(2 \pi t^{*}\right)=Q_{m}^{*} \cos \left(2 \pi t^{*}+\theta\right),
\end{aligned}
$$

where

$$
\begin{gathered}
Q_{m}^{*}=\left(\left(1+\frac{1}{a b} \sum_{m=1}^{\infty} d_{5 m}\right)^{2}+\left(\frac{1}{a b} \sum_{m=1}^{\infty} d_{6 m}\right)^{2}\right)^{1 / 2}, \\
\theta=\arctan \left(\frac{\left((1 / a b) \sum_{m=1}^{\infty} d_{6 m}\right)}{\left(1+(1 / a b) \sum_{m=1}^{\infty} d_{5 m}\right)}\right) .
\end{gathered}
$$

In this study, we analyze the flow pattern through the rectangular microchannel having the same size of the crosssectional area of $1.0 \times 10^{-6} \mathrm{~m}^{2}$ for $\rho=1060 \mathrm{~kg} / \mathrm{m}^{3}, \mu=$ $10^{-3} \mathrm{~Pa} \cdot \mathrm{s}, a_{0}=1, c_{0}=1$, and $b_{1}=1$.

For Case 1, as the pressure gradient is constant, the normalized velocity and flow rate as well as shear stress vary with the slip length and the geometry of the cross-section, namely, the ratio $\varepsilon=b / a$ of the cross-sectional area. The influences of the slip length and the ratio $\varepsilon$ on the flow behavior are demonstrated by analyzing the solutions graphically. Figures 3 and 4 show, respectively, the three-dimensional and two-dimensional velocity profiles on a cross-section of the channel obtained from (39) for four different values of the slip length, $\ell=0.001,0.002,0.003,0.004$. The results indicate that the axial velocity increases significantly when the slip length increases. Figure 5 shows the effects of the slip length and ratio $\varepsilon$ on the flow rate $Q_{0}^{*}$. It is found that the flow rate is linear in slip length. For the same size of the cross-sectional area with the variation of the ratio $\varepsilon$, the flow rate increases significantly as the ratio increases.

For Case 2, as the pressure gradient depends on time, the normalized velocity and flow rate as well as shear stress vary with time and the slip length. We investigate the axial velocity $u^{*}$ on a cross-section of the channel having the ratio $\varepsilon$ of 1 and the slip length of 0.001 under the frequency $\omega=\alpha / \rho a^{2}$ with $\alpha=0.005$ at various instants of time. Figures 6 and 7 show the transient velocity $u^{*}$ obtained from (43) for the slip length $\ell=$ 0.001 . For $t^{*}=0$, the axial velocity has similar profile with $t^{*}=1$, as $e^{i 2 \pi t^{*}}=1$ is constant on the velocity equation (43) so that the curves coincide. The influences of the slip length, the frequency $\omega$, and the ratio $\varepsilon$ of the cross-section of the channel on the flow behavior are illustrated by analyzing the solutions graphically. Figure 8 shows the transient flow rate $Q_{1}^{*}$ on a cross-section of the channel for four different values of the slip length, $\ell=0.001,0.002,0.003,0.004$. Figure 9 presents the influence of $\ell$ on the amplitude $Q_{m}^{*}$ of the flow rate for various frequencies $\omega=\alpha / \rho a^{2}$ with four different $\alpha$

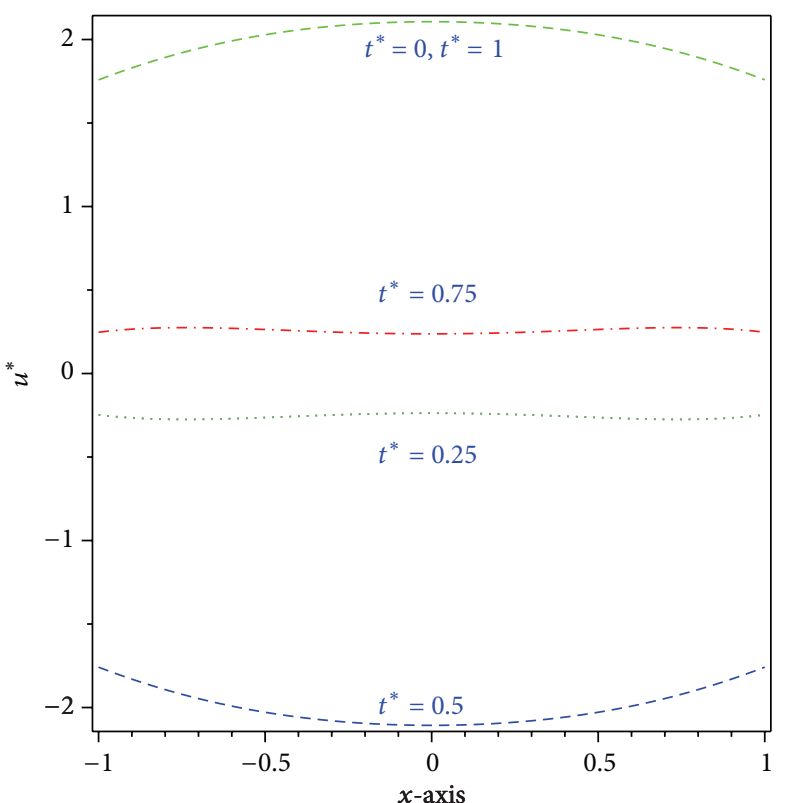

(a)

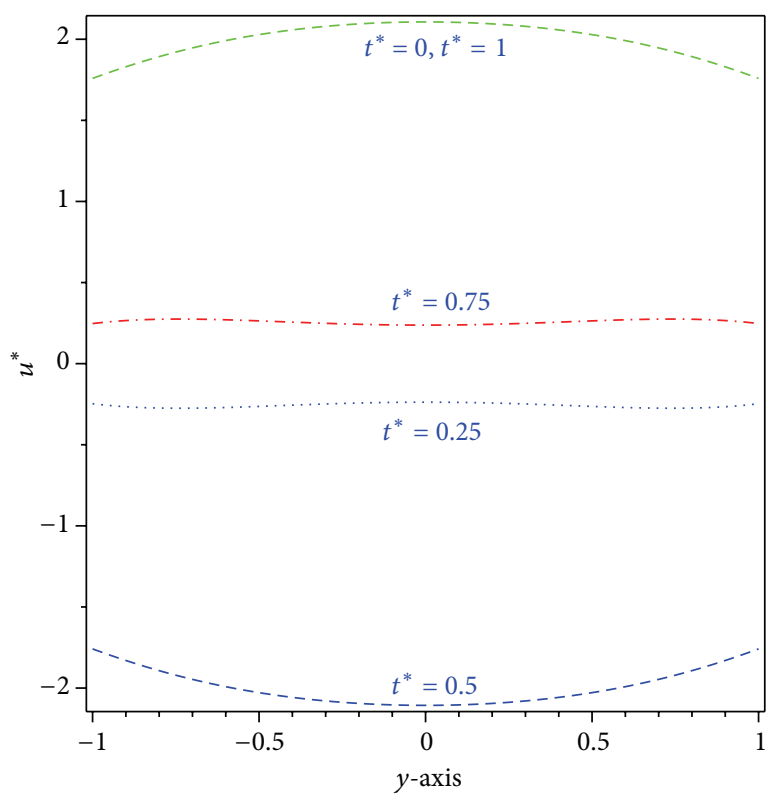

(b)

FIgURE 7: Axial velocity along the $x$-axis and $y$-axis on the crosssection of the channel with the area of $1.0 \times 10^{-6} \mathrm{~m}^{2}$ and ratio $b / a=$ 1 under the frequency $\omega=\alpha / \rho a^{2}$ with $\alpha=0.005$ and slip length of 0.001 at various instants of time.

values: $\alpha=0.0001,0.0005,0.001,0.005$. The result indicates that the dependence of $Q_{m}^{*}$ on $\ell$ is different for different $\omega$. At high frequency (high $\alpha$ ), the amplitude of the flow rate increases initially as $\ell$ increases but it then tends to a constant value once the slip length becomes sufficiently large. Figure 10 shows the influence of the ratio $\varepsilon$ under the same crosssectional area size on the flow rate for different slip lengths $\ell$. It is noted that as the ratio $\varepsilon$ increases the flow rate increases but tends to a constant value as $\varepsilon$ becomes sufficiently large. 


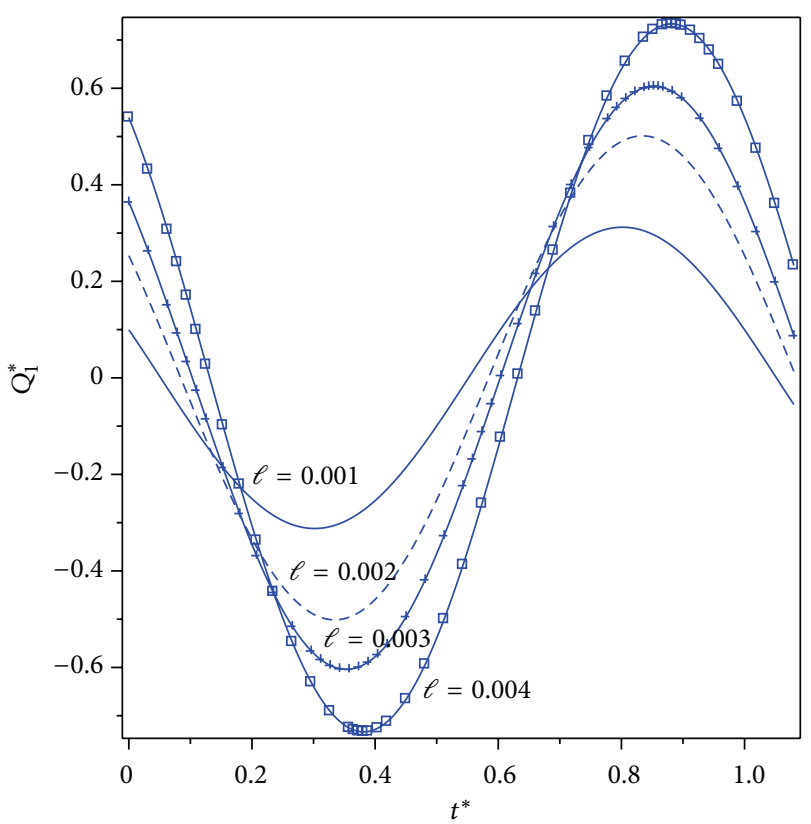

FIGURE 8: Influence of slip length on the flow rate $Q_{1}^{*}$ on the crosssection of the channel with the area of $1.0 \times 10^{-6} \mathrm{~m}^{2}$ and ratio $b / a$ of 1 under the frequency $\omega=\alpha / \rho a^{2}$ with $\alpha=0.005$ at various instants of time.

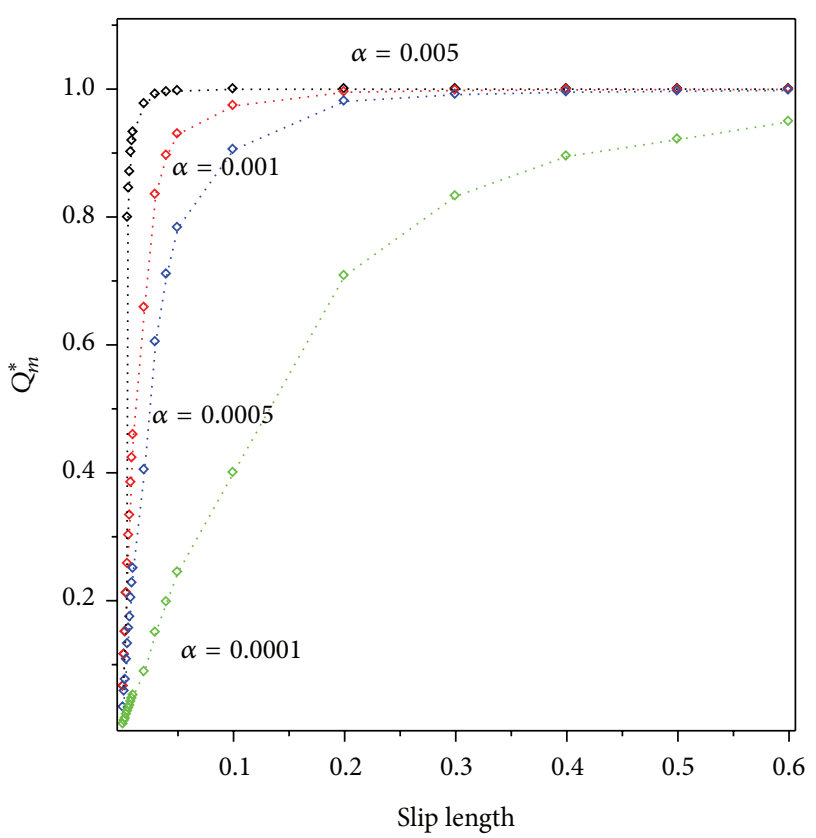

FIGURE 9: Influence of slip length on the amplitude of the flow rate $Q_{m}^{*}$ on the cross-section of the channel with the area of $1.0 \times 10^{-6} \mathrm{~m}^{2}$ and ratio $b / a$ of 1 under different frequencies $\omega=\alpha / \rho a^{2}$ with different $\alpha$.

\section{Conclusions}

In this paper, we derive an exact solution for the unsteady flow of an incompressible Newtonian fluid in a rectangular microchannel with a Navier-slip boundary. From the explicit

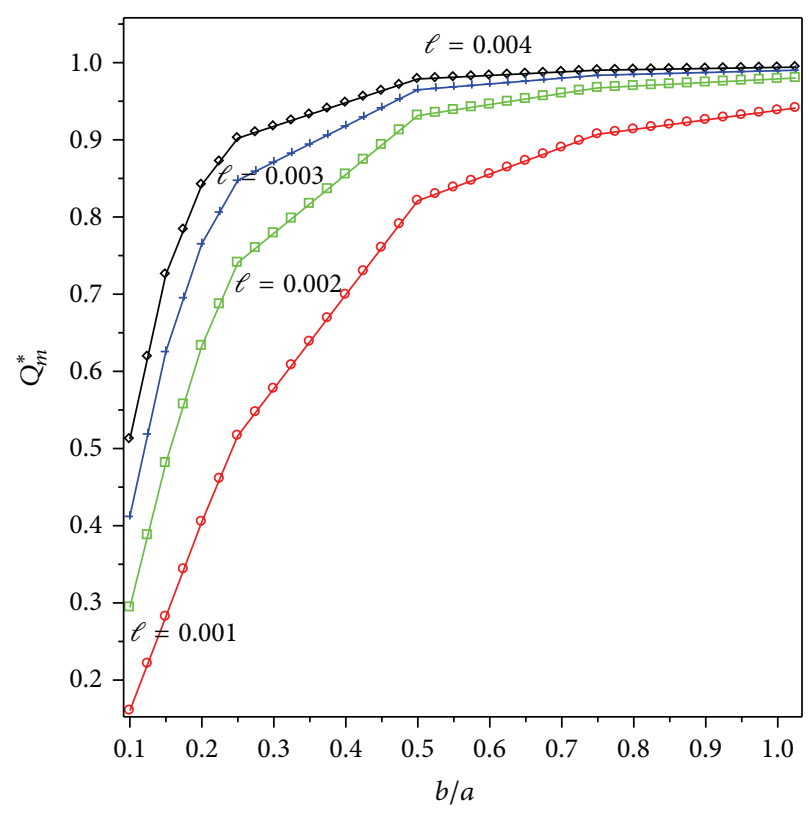

Figure 10: Influence of ratio $\varepsilon$ of $b / a$ and slip length $\ell$ on the amplitude of the flow rate on the cross-section of the channel with constant area of $1.0 \times 10^{-6} \mathrm{~m}^{2}$ under the frequency $\omega=\alpha / \rho a^{2}$ with $\alpha=0.005$.

analytical solutions of the velocity and flow rate, we investigate the effect of the slip length $\ell$ and the geometry of the cross-section on the flow of the fluid through the channel. The investigation shows the following.

(1) For the flow through rectangular microchannels with constant pressure gradient, the axial velocity increases faster in the center of the cross-section than in other areas as the slip length increases, while for the flows, due to the waveform pressure gradient, the velocity changes significantly as the slip length increases.

(2) For flow driven by a constant pressure gradient, the flow rate is linear with respect to the slip length and the ratio $(\varepsilon=b / a)$, and, for different values of $\varepsilon$ with constant $(a \times b)$, the flow rate increases when $\varepsilon$ increases and also when the slip length increases.

(3) The amplitudes of flow rate initially increase significantly as slip length $\ell$ increases but tend to a constant value when $\ell$ becomes sufficiently large for various $\alpha$ values. This profile is similar to the case shown in [39].

\section{Conflict of Interests}

The authors declare that there is no conflict of interests regarding the publication of this paper.

\section{Acknowledgments}

The authors wish to acknowledge the support of the Faculty of Science, Mahidol University. The second author gratefully acknowledges the support of the Australian Research Council through a discovery project grant. The authors are also 
grateful to both referees for their comments and suggestions which have led to significant improvement of this paper.

\section{References}

[1] R. B. Bird, W. E. Stewart, and E. N. Lightfoot, Transport Phenomena, John Wiley \& Sons, New York, NY, USA, 1960.

[2] P. Huang and K. S. Breuer, "Direct measurement of slip length in electrolyte solutions," Physics of Fluids, vol. 19, no. 2, Article ID 028104, 2007.

[3] D. You and P. Moin, "Effects of hydrophobic surfaces on the drag and lift of a circular cylinder," Physics of Fluids, vol. 19, no. 8, Article ID 081701, 2007.

[4] M. Gad-el-Hak, "The fluid mechanics of microdevices-the Freeman scholar lecture," Journal of Fluids Engineering, vol. 121, no. 1, pp. 5-33, 1999.

[5] C. Ho and Y. Tai, "Micro-electro-mechanical-systems (MEMS) and fluid flows," Annual Review of Fluid Mechanics, vol. 30, pp. 579-612, 1998.

[6] H. Huang, T. S. Lee, and C. Shu, "Lattice Boltzmann method simulation gas slip flow in long microtubes," International Journal of Numerical Methods for Heat \& Fluid Flow, vol. 17, no. 6, pp. 587-607, 2007.

[7] F. Saidi, "Non-Newtonian flow in a thin film with boundary conditions of Coulomb's type," Zeitschrift für Angewandte Mathematik und Mechanik, vol. 86, no. 9, pp. 702-721, 2006.

[8] S. J. Liao, Beyond Perturbation: Introduction to Homotopy Analysis Method, Chapman \& Hall/CRC Press, Boca Raton, Fla, USA, 2003.

[9] H. Herwig and O. Hausner, "Critical view on "new results in micro-fluid mechanics": an example," International Journal of Heat and Mass Transfer, vol. 46, no. 5, pp. 935-937, 2003.

[10] B. Wiwatanapataphee, D. Poltem, Y. H. Wu, and Y. Lenbury, "Simulation of pulsatile flow of blood in stenosed coronary artery bypass with graft," Mathematical Biosciences and Engineering, vol. 3, no. 2, pp. 371-383, 2006.

[11] T. C. Papanastasiou, "Flows of materials with yield," Journal of Rheology, vol. 31, no. 5, pp. 385-404, 1987.

[12] J. P. Pascal, "Instability of power-law fluid flow down a porous incline," Journal of Non-Newtonian Fluid Mechanics, vol. 133, no. 2-3, pp. 109-120, 2006.

[13] R. Pit, H. Hervet, and L. Léger, "Direct experimental evidence of slip in hexadecane: solid interfaces," Physical Review Letters, vol. 85, no. 5, pp. 980-983, 2000.

[14] P. A. Thompson and S. M. Troian, "A general boundary condition for liquid flow at solid surfaces," Nature, vol. 389, no. 6649, pp. 360-362, 1997.

[15] G. Tunc and Y. Bayazitoglu, "Heat transfer in rectangular microchannels," International Journal of Heat and Mass Transfer, vol. 45, no. 4, pp. 765-773, 2002.

[16] C. Y. Wang, "Exact solutions of the unsteady Navier-Stokes equations," Applied Mechanics Reviews, vol. 42, no. 11, pp. S269S282, 1989.

[17] B. Wiwatanapataphee, Y. H. Wu, M. Hu, and K. Chayantrakon, "A study of transient flows of Newtonian fluids through microannuals with a slip boundary," Journal of Physics A: Mathematical and Theoretical, vol. 42, no. 6, Article ID 065206, 2009.

[18] A. M. Afonso, L. L. Ferrás, J. M. Nóbrega, M. A. Alves, and F. T. Pinho, "Pressuredriven electrokinetic slip flows of viscoelastic fluids in hydrophobic microchannels," Microfluidics and Nanofluidics, vol. 26, pp. 1-12, 2013.
[19] S. R. Deshmukh and D. G. Vlachos, "CFD simulations of coupled, countercurrent combustor/reformer microdevices for hydrogen production," Industrial and Engineering Chemistry Research, vol. 44, no. 14, pp. 4982-4992, 2005.

[20] Z. Duan and B. He, "Discussion: slip-flow in microchannels of non-circular cross sections," Journal of Fluids Engineering, vol. 136, no. 5, 2014.

[21] M. Fichman and G. Hetsroni, "Viscosity and slip velocity in gas flow in microchannels," Physics of Fluids, vol. 17, no. 12, Article ID 123102, pp. 1-5, 2005.

[22] A. He, "Interfacial instability of compressible slip flows in a microchannel," Physical Review E, vol. 87, no. 5, Article ID 053006, 2013.

[23] T. Kuo, D. M. Cannon Jr., M. A. Shannon, P. W. Bohn, and J. V. Sweedler, "Hybrid three-dimensional nanofluidic/microfluidic devices using molecular gates," Sensors and Actuators, A: Physical, vol. 102, no. 3, pp. 223-233, 2003.

[24] H. B. Lee, I. W. Yeo, and K. K. Lee, "Water flow and slip on NAPL-wetted surfaces of a parallel-walled fracture," Geophysical Research Letters, vol. 34, no. 19, Article ID L19401, 2007.

[25] M. T. Matthews and J. M. Hill, "Newtonian flow with nonlinear Navier boundary condition," Acta Mechanica, vol. 191, no. 3-4, pp. 195-217, 2007.

[26] S. Palle and S. Aliabadi, "Slip flow and heat transfer in rectangular and circular microchannels using hybrid FE/FV method," International Journal for Numerical Methods in Engineering, vol. 89, no. 1, pp. 53-70, 2012.

[27] L. Szalmas, "Slip-flow boundary condition for straight walls in the lattice Boltzmann model," Physical Review E, vol. 73, no. 6, Article ID 066710, 2006.

[28] Q. Sun, Y. H. Wu, L. Liu, and B. Wiwatanapataphee, "Study of a Newtonian fluid through circular channels with slip boundary taking into account electrokinetic effect," Abstract and Applied Analysis, vol. 2013, Article ID 718603, 9 pages, 2013.

[29] F. Tahmouresi and S. K. Das, "Analytical modeling of gaseous slip flow in parabolic microchannels," Journal of Fluids Engineering, vol. 136, no. 7, pp. 10-1115, 2014.

[30] A. Tamayol and K. Hooman, "Slip-flow in microchannels of non-circular cross sections," Journal of Fluids Engineering, vol. 133, no. 9, Article ID 091202, 2011.

[31] G. H. Tang, X. F. Li, Y. L. He, and W. Q. Tao, "Electroosmotic flow of non-Newtonian fluid in microchannels," Journal of NonNewtonian Fluid Mechanics, vol. 157, no. 1-2, pp. 133-137, 2009.

[32] Y. H. Wu, B. Wiwatanapataphee, and M. Hu, "Pressure-driven transient flows of Newtonian fluids through microtubes with slip boundary," Physica A: Statistical Mechanics and Its Applications, vol. 387, no. 24, pp. 5979-5990, 2008.

[33] C. Zhang, Y. Chen, Z. Deng, and M. Shi, "Role of rough surface topography on gas slip flow in microchannels," Physical Review E, vol. 86, no. 1, Article ID 016319, 2012.

[34] Z. Duan and Y. S. Muzychka, "Slip flow in elliptic microchannels," International Journal of Thermal Sciences, vol. 46, no. 11, pp. 1104-1111, 2007.

[35] Z. Duan and Y. S. Muzychka, "Slip flow in non-circular microchannels," Microfluidics and Nanofluidics, vol. 3, no. 4, pp. 473-484, 2007.

[36] S. C. Hunter, Mechanics of Continuous Media, John Wiley \& Sons, New York, NY, USA, 1983.

[37] T. R. Salamon, D. E. Bornside, R. C. Armstrong, and R. A. Brown, "Local similarity solutions in the presence of a slip boundary condition," Physics of Fluids, vol. 9, no. 5, pp. 12351247, 1997. 
[38] E. B. Arkilic, "Slip flow in microchannel," in Proceeding of Rarefield Gas Dynamic Symposium, Oxford, UK, July 1994.

[39] M. Bahrami, M. M. Yovanovich, and J. R. Culham, "Pressure drop of fully-developed, laminar flow in microchannel of arbitrary cross-section," Journal of Fluids Engineering, vol. 128, no. 5, pp. 1036-1044, 2006.

[40] R. Baviere, F. Ayela, S. le Person, and M. Favre-Marinet, "Experimental characterization of water flow through smooth rectangular microchannels," Physics of Fluids, vol. 17, no. 9, Article ID 098105, 2005.

[41] G. Chakraborty, "A note on methods for analysis of flow through microchannels," International Journal of Heat and Mass Transfer, vol. 51, no. 17-18, pp. 4583-4588, 2008.

[42] W. A. Ebert and E. M. Sparrow, "Slip flow in rctangular and annular ducts," Journal of Fluids Engineering, vol. 87, no. 4, pp. 1018-1024, 1965.

[43] V. O'Brien, "Pulsatile fully developed flow in rectangular channels," Journal of the Franklin Institute, vol. 800, no. 3, pp. 225230, 1975.

[44] C. Aubert and S. Colin, "High-order boundary conditions for gaseous flows in rectangular microducts," Microscale Thermophysical Engineering, vol. 5, no. 1, pp. 41-54, 2001.

[45] E. Lauga and T. M. Squires, "Brownian motion near a partialslip boundary: a local probe of the no-slip condition," Physics of Fluids, vol. 17, no. 10, Article ID 103102, 2005.

[46] E. Lauga and C. Cossu, "A note on the stability of slip channel flows," Physics of Fluids, vol. 17, no. 8, pp. 1-4, 2005.

[47] G. L. Morini and M. Spiga, "Slip flow in rectangular microtubes," Microscale Thermophysical Engineering, vol. 2, no. 4, pp. 273282, 1998.

[48] M. Spiga and G. L. Morini, "Laminar heat transfer in rectangular ducts," Trends in Heat, Mass \& Momentum Transfer, vol. 3, pp. 19-30, 1997. 


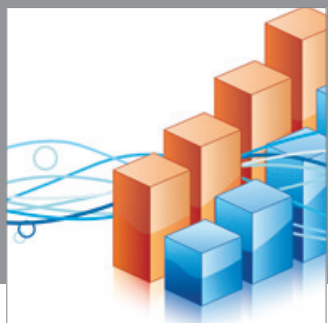

Advances in

Operations Research

mansans

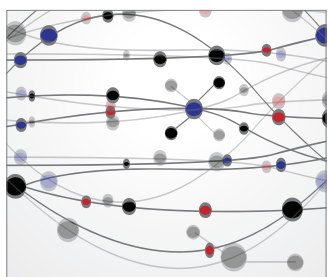

The Scientific World Journal
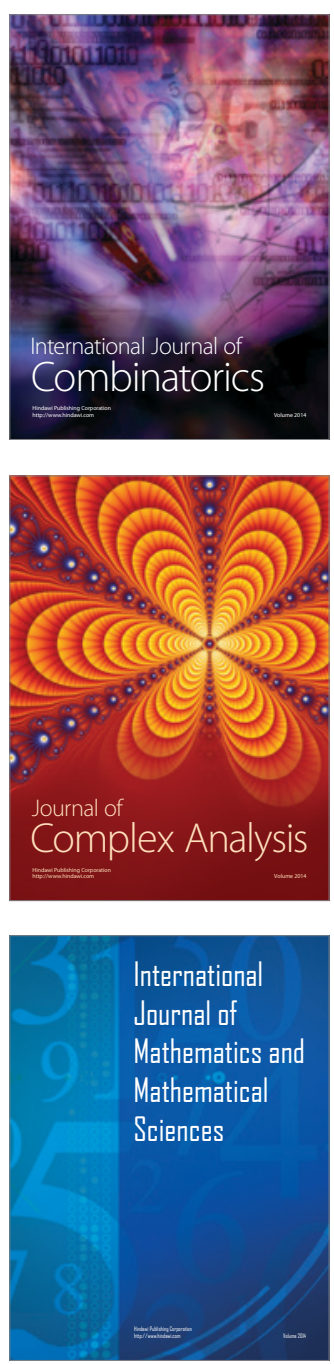
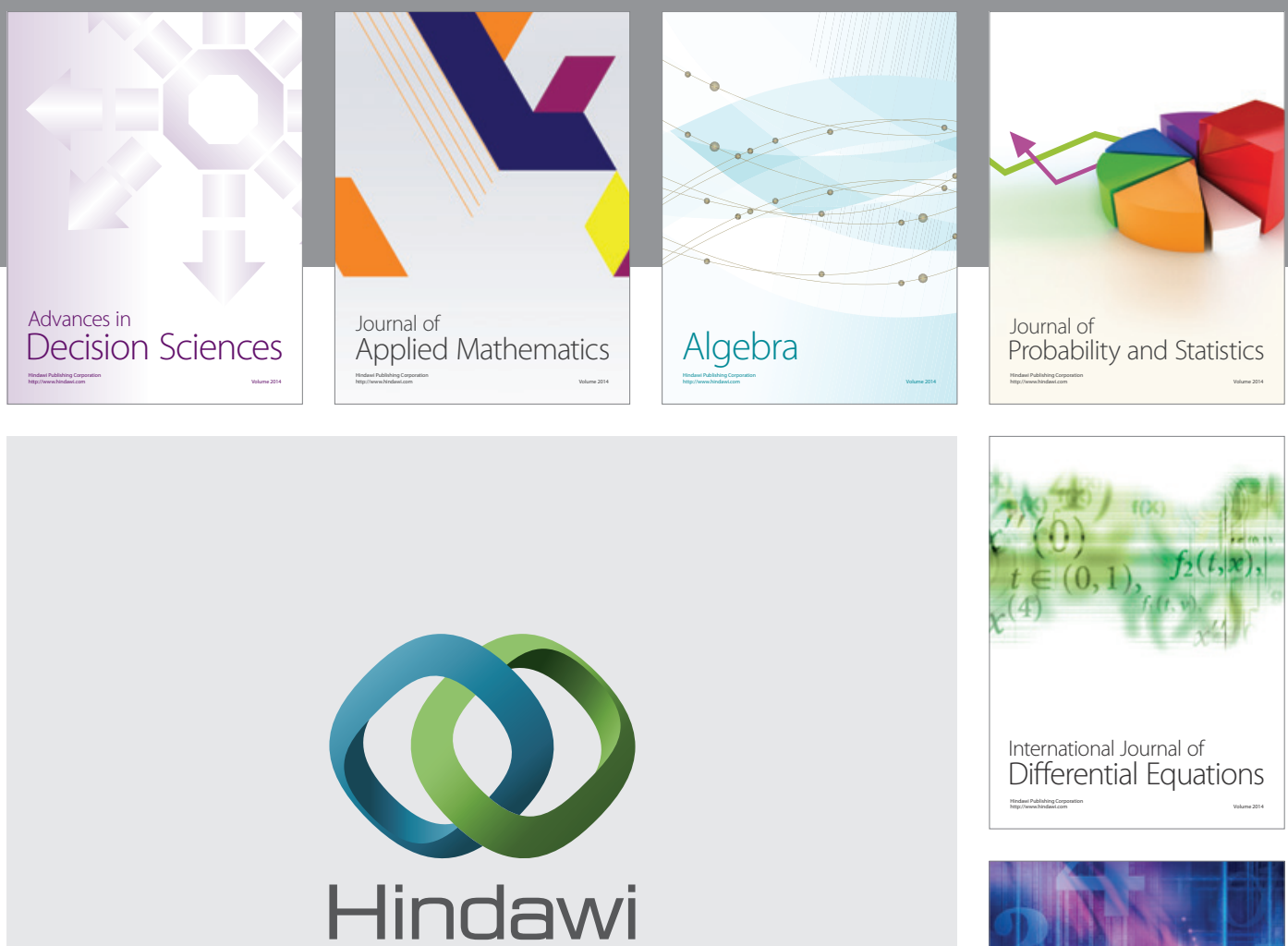

Submit your manuscripts at http://www.hindawi.com
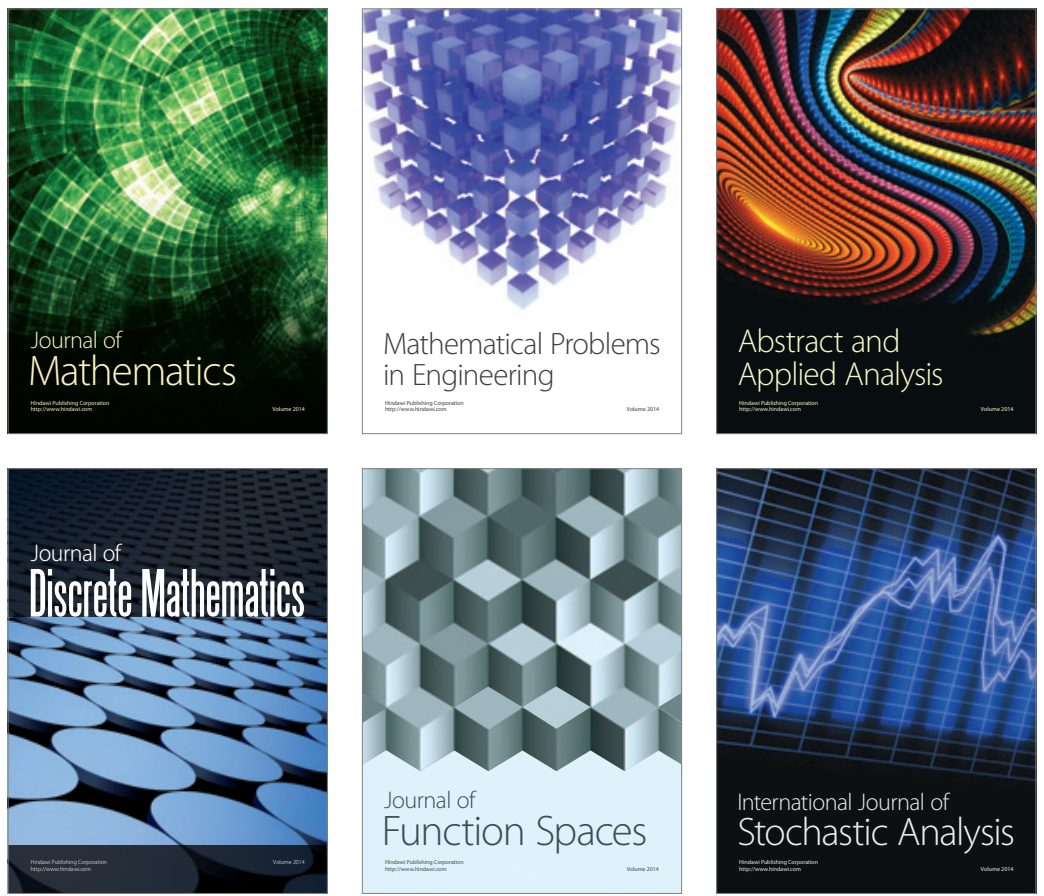

Journal of

Function Spaces

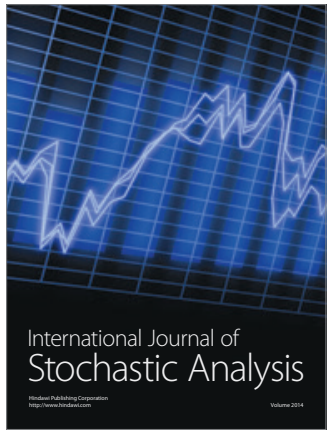

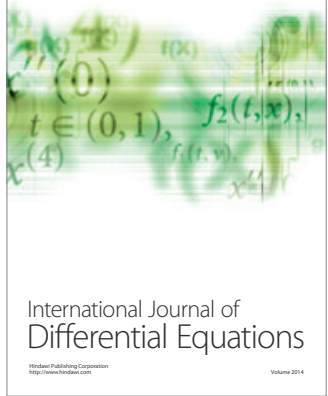
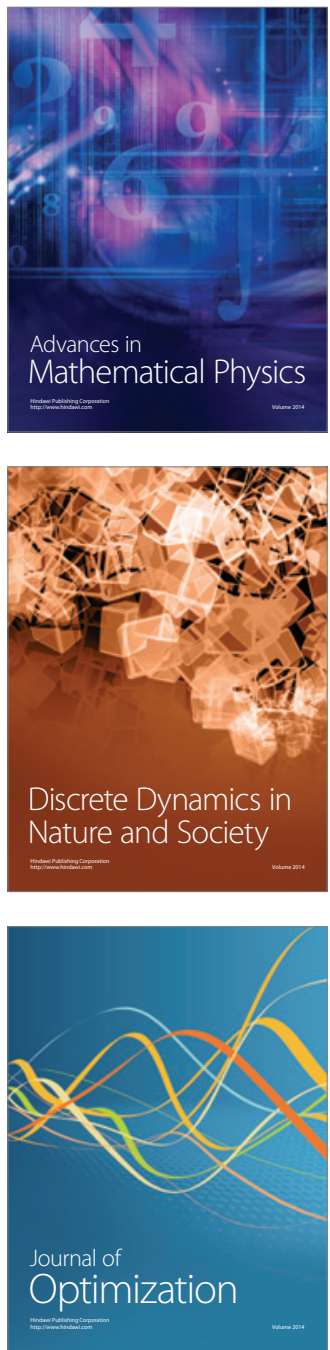